Full Length Article

\title{
Low-temperature growth and electronic structures of ambipolar Yb-doped zinc tin oxide transparent thin films
}

\author{
Seol Hee Oh ${ }^{\mathrm{a}}$, Gerald Ferblantier ${ }^{\mathrm{b}}$, Young Sang Park ${ }^{\mathrm{b}}$, Guy Schmerber ${ }^{\mathrm{c}}$, Aziz Dinia ${ }^{\mathrm{c}}$, Abdelilah Slaoui $^{\mathrm{b}}$, \\ William Jo ${ }^{\text {a,* }}$ \\ a Department of Physics, Ewha Womans University, Seoul 03760, Republic of Korea \\ ${ }^{\mathrm{b}}$ ICube, CNRS-Université de Strasbourg, UMR 7357, 23 rue du Loess, BP 20 CR, 67037 Strasbourg Cedex 2, France \\ ' IPCMS, CNRS-Université de Strasbourg, UMR 7504, 23 rue du Loess, BP 43, 67034 Strasbourg Cedex 2, France
}

\section{A R T I C L E I N F O}

Article history:

Received 13 November 2017

Revised 16 January 2018

Accepted 1 February 2018

Available online 3 February 2018

Keywords:

Transparent conducting oxide

Zinc tin oxides

Amorphous oxide thin films

Kelvin probe force microscopy

Hall measurement

Compositional dependence

\begin{abstract}
A B S T R A C T
The compositional dependence of the crystal structure, optical transmittance, and surface electric properties of the zinc tin oxide (Zn-Sn-O, shortened ZTO) thin films were investigated. ZTO thin films with different compositional ratios were fabricated on glass and p-silicon wafers using radio frequency magnetron sputtering. The binding energy of amorphous ZTO thin films was examined by a X-ray photoelectron spectroscopy. The optical transmittance over 70\% in the visible region for all the ZTO films was observed. The optical band gap of the ZTO films was changed as a result of the competition between the Burstein-Moss effect and renormalization. An electron concentration in the films and surface work function distribution were measured by a Hall measurement and Kelvin probe force microscopy, respectively. The mobility of the n- and p-type ZTO thin films have more than $130 \mathrm{~cm}^{2} / \mathrm{V} \mathrm{s}$ and $15 \mathrm{~cm}^{2} / \mathrm{V} \mathrm{s}$, respectively. We finally constructed the band structure which contains band gap, work function, and band edges such as valence band maximum and conduction band minimum of ZTO thin films. The present study results suggest that the ZTO thin film is competitive compared with the indium tin oxide, which is a representative material of the transparent conducting oxides, regarding optoelectronic devices applications.
\end{abstract}

(c) 2018 Elsevier B.V. All rights reserved.

\section{Introduction}

The band gap of the transparent conducting oxide (TCO) films is a wider than $\sim 3 \mathrm{eV}$ and can be used for the transmission of the visible light and low resistivity from doped charge carriers. Doping with impurities, mainly rare-earth elements, into the transparent oxide attributes both of the high conductivity and the stability at high temperature. TCOs have been used in many applications including the transparent electrode in flat-panel optoelectronic devices such as solar cells, liquid-crystal displays, and lightemitting diodes [1-3]. The focus of the majority of the TCO research has been $\mathrm{Sn}$ doped $\mathrm{In}_{2} \mathrm{O}_{3}$ (ITO), and it has been common to apply transparent electrodes. However, the finding of an alternative material has been necessary due to the high cost and toxic nature of the indium in ITO. Here, an amorphous multicomponent oxide Zn-Sn-O (ZTO) system has attracted much attention as a new alternative to ITO materials, since it is inexpensive compared to TCO materials including rare earth metals and do not contain

\footnotetext{
* Corresponding author.

E-mail address: wmjo@ewha.ac.kr (W. Jo).
}

toxic elements [4,5]. Furthermore, ZTO films can have smooth and hard surface morphology. Hence, they are suitable for barrier layers for electronic devices or optical products because they exhibit good thermal and chemical stabilities [6]. ZTO films show a different crystal structure with respect to the ratio of $\mathrm{Zn}$ and $\mathrm{Sn}$. Crystalline $\mathrm{ZnSnO}_{3}$ and $\mathrm{Zn}_{2} \mathrm{SnO}_{4}$ have the ilmenite and the inverse spinel structure, respectively $[7,8]$. ZTO films in elemental composition can have an amorphous structure which can be fabricated at low substrate temperature, even room temperature [9]. Inherent advantages exist regarding the amorphous TCO films for applications such as organic light emitting diodes and thin film transistor-liquid crystal displays. Zinc oxide ( $\mathrm{ZnO})$ and tin oxide $\left(\mathrm{SnO}_{2}\right)$-based TCO films are easily doped as n-type semiconductors, but it is also necessary to obtain p-type TCOs, which are difficult to grow because of the self-compensating native donor defects such as the oxygen vacancies and the interstitials in the TCO structure $[10,11]$. In principle, the p-type conductivity can be realized by doping with the group-IV element in the plane of the $\mathrm{Zn}$ site in the ZTO system [12,13]. In the p-type TCO film, compositional control is one of the most important issues, because a specific physical condition has been required for specialized applications. In this 
study, the effect of the Sn concentration on the electrical and optical properties of amorphous ZTO films is reported. The ZTO films with the different $S n /(Z n+S n)$ compositional ratios were used to investigate the tunable physical properties for optoelectronic applications.

\section{Method}

The ZTO thin films were deposited onto glass and p-silicon substrates at $300{ }^{\circ} \mathrm{C}$ with zinc and tin metallic targets in an Orion $3 \mathrm{RF}$ (Radio-Frequency) reactive magnetron sputtering system from AJA International Co. A Zn target covered with small discs of pure $\mathrm{Yb}$ was used. Argon and oxygen gas were introduced in the sputtering chamber as reactive gases to deposit the $\mathrm{Yb}: \mathrm{ZnSnO}_{\mathrm{x}}$ films. For all depositions, the targetsubstrate distance was kept at about $12 \mathrm{~cm}$; the working pressure was set at $3.4 \mathrm{mTorr}$. The substrate holder was rotating in order to insure an improved homogeneity of the layers. The base pressure is $1 \times 10^{-8} \mathrm{mbar}$, and the argon ( $\mathrm{Ar}$ ) and oxygen $\left(\mathrm{O}_{2}\right)$ gases were introduced into the sputtering chamber with constant flow rates of $8 \mathrm{sccm}$ for the $\mathrm{Ar}$ and $3 \mathrm{sccm}$ for the $\mathrm{O}_{2}$ using a standard mass flow controller. The RF power of the Sn varied from 10 to $70 \mathrm{~W}$ with a constant $\mathrm{Zn}: \mathrm{Yb}$ power of $70 \mathrm{~W}$. The film thickness and the cationic composition of the ZTO thin films, which are shown in Table 1, were determined using the field-emission scanning electron microscopy (FE-SEM) (S-4200, Hitachi) device equipped with an energy dispersion spectroscopy (EDS) functionality. The crystallographic structure of the ZTO thin film was determined using typical $\theta-2 \theta$ scans and grazing incidence X-ray diffraction (XRD) experiments at room temperature using the Rigaku DMAX-2500 device with $\mathrm{Cu}-\mathrm{K}_{\alpha}$ radiation $(0.154 \mathrm{~nm})$ over the range of $10-$ $90^{\circ}$. The surface morphology and the local electrical properties of the ZTO films were examined using the Kelvin probe force microscopy (KPFM) (n-Tracer, Nanofocus) using Pt/Ir-coated Si atomic force microscopy (AFM) tip with a resonant frequency of $71-72 \mathrm{kHz}$. The WSxM software provided support in the KPFM analysis.

The carrier concentration was obtained from the Hall effect measurement (model HMS-5300, Ecopia) using the Van der Pauw method with a constant magnetic field of 0.5 Tesla at room temperature. The optical transmittance was measured with a Lambda 950 Perkin-Elmer UV-visible-NIR (UV-Vis-NIR) spectrophotometer in the wavelength range of $250-1500 \mathrm{~nm}$ [14]. The measurements using the X-ray photoelectron spectroscopy (XPS) (AXIS His, Kratos) were conducted under a base pressure below $10^{-8}$ Torr, with a monochromatized $\mathrm{Al} \mathrm{K} \mathrm{K}_{\alpha}$ radiation $(\mathrm{h} v=1486 \mathrm{eV})$ and at a constant dwelling time of $100 \mathrm{~ms}$ and a flood gun was operated at a $1 \mathrm{eV}$ energy and an anode current of $0.1 \mathrm{~mA}$. All XPS peaks were calibrated using the $\mathrm{C} 1 \mathrm{~s}$ binding energy of $284.6 \mathrm{eV}$ as a reference. The elemental composition was determined by calculating the relative XPS peak intensities at the specific binding energies of the $\mathrm{Zn} 2 \mathrm{p}$ and $\mathrm{Sn} 3 \mathrm{~d}$ states.

\section{Results and discussion}

The $\theta-2 \theta$ XRD patterns versus the Sn contents in the ZTO films that were deposited on the Si (100) substrate are shown in Fig. 1(a). Except for the Sn10 sample, the XRD profiles of the ZTO films do not show a crystalline structure. In the case of the Sn10 film, the XRD peaks at $2 \theta=33.9^{\circ}$ and $71.3^{\circ}$ can be attributed to the (002) and (004) reflections of $\mathrm{ZnO}(00 \mathrm{l})$ structure. The grazing incident XRD was also measured to confirm the amorphous phase of the ZTO films, because the asymmetric geometry of the grazing incident XRD measurement could exclude the substrate signal, while the geometry of the $\theta-2 \theta$ XRD measurement is symmetric geometry and was used to determine the normal plane crystalline structure. The $\mathrm{ZnO}$ phase in the Sn10 film was also detected through the use of a grazing incident XRD pattern, while a phase is not present in the Sn30 film, as shown in Fig. 1(b). The amorphous phase of the ZTO thin films is attributed to the high Sn concentration. The report indicates that the Sn-doped $\mathrm{ZnO}$ thin films were decrystallized with the increasing of the Sn concentration from 0 to 5 at.\% [15]. The proposed ZTO thin films, except Sn10, contain concentrations that are more than 15 at.\% which is a relatively high status compared with the previous work.

The XPS analysis was performed on the ZTO thin films to determine the variations of the chemical states of the $\mathrm{Zn}$ and the Sn. Fig. 2 shows the $\mathrm{Zn} 2 \mathrm{p}$ and Sn 3d XPS spectra of the amorphous ZTO thin films with different Sn concentrations. The XPS spectra were obtained after the pre-cleaning of the sample surface to prevent the contamination of the films. The $C 1 \mathrm{~s}$ with a binding energy of $284.6 \mathrm{eV}$ were taken as a reference to calibrate the binding energies [16]. In Fig. 2(a), the XPS peaks that are evident at around $1021.4 \mathrm{eV}$ and $1044.5 \mathrm{eV}$ and correspond to the binding energies of the $\mathrm{Zn} 2 \mathrm{p}_{3 / 2}$ and $\mathrm{Zn} 2 \mathrm{p}_{1 / 2}$ levels, respectively, are consistent with the $\mathrm{Zn}^{2+}$ ion binding in the ZTO films $[17,18]$. It is notable that no significant XPS signals were observed for $\mathrm{Yb}$ binding states in the whole measurement range. Influence of $\mathrm{Yb}$ on physical properties of ZTO films is negligible, if any. The intensities of the XPS peaks of $Z n 2 p_{3 / 2}$ and $Z n 2 p_{1 / 2}$ were reduced with the increase of $S n$ concentration. Furthermore, the $\mathrm{Sn} 3 \mathrm{~d}_{5 / 2}$ and $\mathrm{Sn} 3 \mathrm{~d}_{3 / 2}$ peaks in Fig. 2 (b) are located at $485.4 \mathrm{eV}$ and $497.1 \mathrm{eV}$, respectively, which can be attributed to the $\mathrm{Sn}^{4+}$ ions in the ZTO film that resulted from the $\mathrm{SnO}_{2}$ phase [19]. As expected, the trends for the XPS peaks of Sn appear contrary to the Zn's case, the intensity of the XPS peaks of $\mathrm{Sn} 3 \mathrm{~d}_{5 / 2}$ and $3 \mathrm{~d}_{3 / 2}$ increased with the increase of Sn concentration. In addition, XPS peaks of $\mathrm{O} 1 \mathrm{~s}$ were observed at 530$533 \mathrm{eV}$ and could be divided into two different peaks. One peak observed at $531 \mathrm{eV}$ corresponds to the binding energy of photoelectrons coming from $\mathrm{O}$ with $\mathrm{Zn}^{2+}$ and $\mathrm{Sn}^{4+}[20,21]$. The binding energies of photoelectrons from $\mathrm{O}-\mathrm{Zn}^{2+}$ and $\mathrm{O}-\mathrm{Sn}^{4+}$ are not distinguishable regardless of crystalline or amorphous ZTO films [22]. The other peak observed at $532.5 \mathrm{eV}$ is attributed to chemisorbed oxygen at grain boundaries or loosely bound oxygen at the surface of the films $[20,21]$.

Table 1

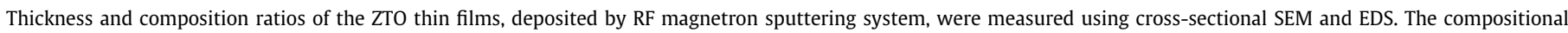
ratios of the right side of the table has been defined as the Sn contents over the total cation contents.

\begin{tabular}{|c|c|c|c|c|}
\hline Sample label & Sn power $[\mathrm{W}]$ & Thickness [nm] & Compositional ratio (Zn:Sn:O:Yb) & $\mathrm{Sn} /(\mathrm{Sn}+\mathrm{Zn}+\mathrm{Yb})[\mathrm{at} . \%]$ \\
\hline Sn10 & 10 & 57.3 & $1.00: 0.00: 4.17: 0.10$ & 0 \\
\hline Sn20 & 20 & 65.6 & $1.00: 0.24: 6.40: 0.14$ & 17.4 \\
\hline Sn30 & 30 & 95.8 & $1.00: 0.56: 6.95: 0.13$ & 33.1 \\
\hline $\operatorname{Sn} 50$ & 50 & 81.3 & $1.00: 2.20: 15.89: 0.35$ & 62.0 \\
\hline Sn60 & 60 & 72.9 & $1.00: 2.55: 21.14: 0.45$ & 63.8 \\
\hline Sn70 & 70 & 88.5 & $1.00: 4.79: 32.20: 0.56$ & 75.4 \\
\hline
\end{tabular}



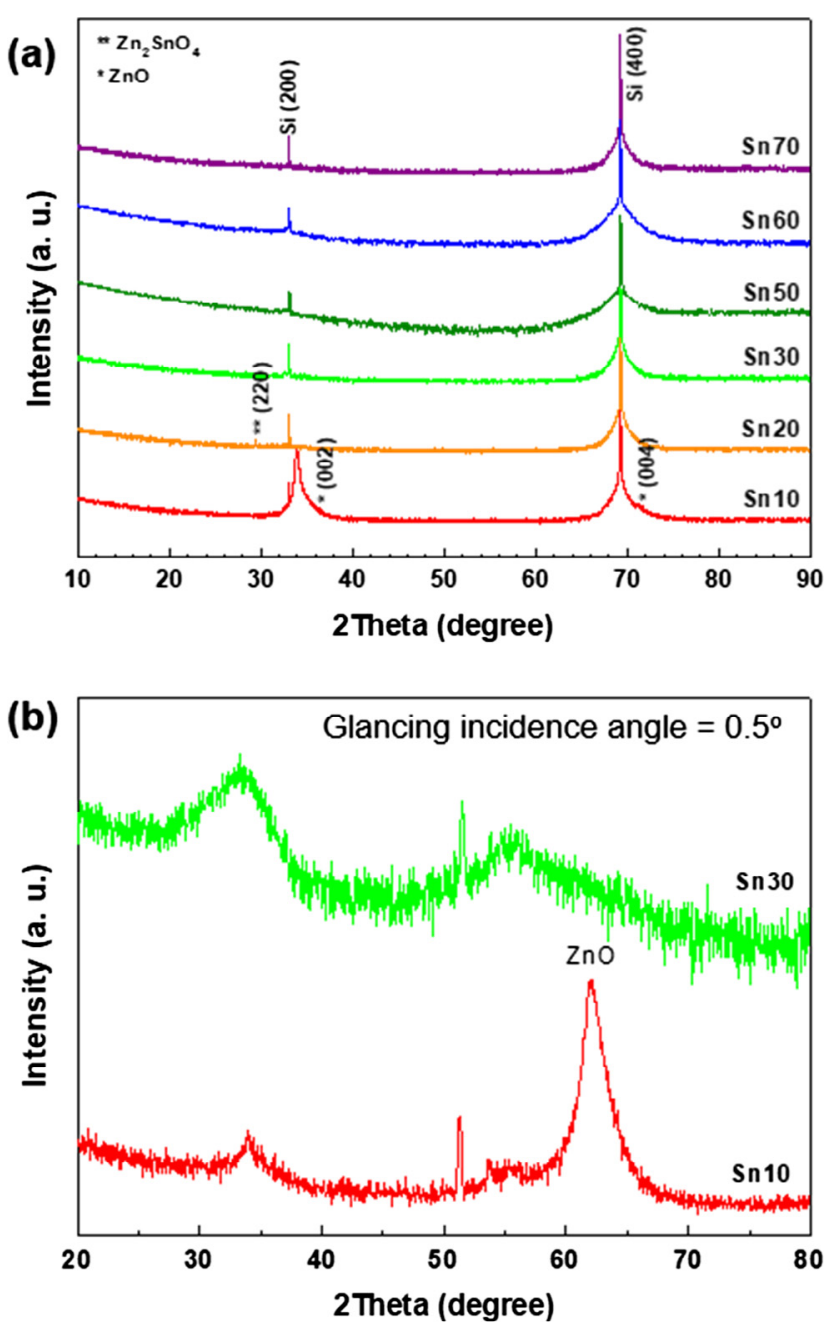

Fig. 1. (a) The $\theta-2 \theta$ XRD patterns of the $\mathrm{ZTO} / \mathrm{Si}(100)$ films with different $\mathrm{Sn}$ contents. (b) Grazing incidence XRD patterns of the Sn10 and the Sn30 where the incidence angle is $0.5^{\circ}$.

The dependence of the Sn concentration on the work functions $(\varphi)$ of the ZTO thin films were measured using KPFM. KPFM is a non-contact electrical force microscopy that uses
AFM, and it is used to measure the two-dimensional image of the contact potential difference $\left(\mathrm{V}_{\mathrm{CPD}}\right)$ between a sample and the conductive AFM tip with a resolution in the nanometer range [23]. Fig. 3(b) shows the $V_{C P D}$ maps of the ZTO films with different $\mathrm{Sn}$ concentration. The $\mathrm{V}_{\mathrm{CPD}}$ images were measured with a constant height after the scanning surface topography that is shown in Fig. 3(a). All of the ZTO films show an even potential distribution without any domain in the KPFM images, and their surfaces are flat with a root mean square (RMS) roughness that is less than $1.5 \mathrm{~nm}$. For computing the work functions from the $\mathrm{V}_{\mathrm{CPD}}$ values, the $\mathrm{V}_{\mathrm{CPD}}$ distributions depending on the Sn concentration in the ZTO film are shown in Fig. 3(c). The work functions of the ZTO films could be calculated using the following equation because the $V_{C P D}$ is equal to the work function difference between the AFM tip and the sample:

$\varphi_{\text {sample }}=-e V_{C P D, H O P G}+\varphi_{H O P G}+e V_{C P D, \text { sample }}$

where $\mathrm{V}_{\mathrm{CPD} \text {,HOPG }}$ and $\mathrm{V}_{\mathrm{CPD} \text {,sample }}$ represent the potential differences regarding the AFM tip potential, and the highly oriented pyrolytic graphite (HOPG) work function $(4.6 \mathrm{eV})$ was used as a reference value. In Fig. 3(d), the work function was reduced with the increasing of the Sn concentration in the ZTO films.

The Hall measurement of the ZTO thin films was performed under a constant magnetic field using a 0.5 Tesla. The Hall coefficient $\left(R_{H}\right)$ and the corresponding carrier concentrations $(n)$, which could be obtained from the $\mathrm{n}$ or $\mathrm{p}=1 / \mathrm{e} R_{\mathrm{H}}$, a dependent on the $\mathrm{Sn}$ concentration, as shown in Fig. 4. For the statistical analysis, the experiment was repeated over 50 times for each sample. For the Sn10 sample, the Hall coefficient was not obtained because of a high resistivity. The ZTO carrier type was changed from the ntype to the p-type at the critical point between the $33.1 \%$ and $62 \%$ Sn concentrations; moreover, the carrier concentrations for all of the ZTO films were increased with the increasing of the Sn concentration. The highest carrier concentration in the ZTO film is $4.73 \times 10^{19} \mathrm{~cm}^{-3}$, where the $\mathrm{Sn}$ at.\% is 33.1 . Such behavior can be explained by a consideration of the element that is dominant in the ZTO film using the following chemical formula with the Kroger-Vink notation [24];

$x \mathrm{ZnO}+y \mathrm{SnO}_{2} \rightarrow x \mathrm{Zn}_{\mathrm{Sn}}^{\prime \prime}+y \mathrm{Sn}_{\mathrm{Zn}}+\left(x+\frac{1}{2} y\right) \mathrm{O}_{2}+2\left(x e^{-}+y h\right)$

Below the critical point, the $\mathrm{ZnO}$ concentration is higher than the $\mathrm{SnO}_{2}$ concentration in the ZTO film, namely $\mathrm{x}>\mathrm{y}$ in Eq. (2).
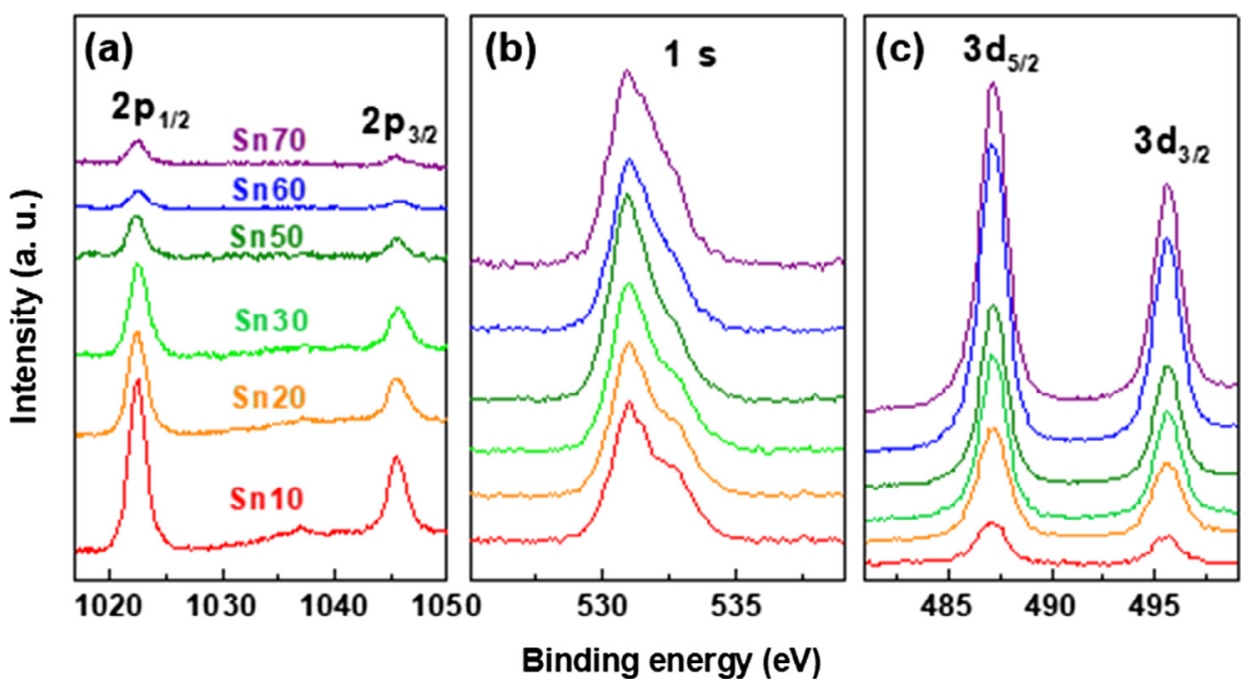

Fig. 2. XPS spectra in ZTO thin films for the following levels: (a) Zn 2p, (b) O 1s, and (c) Sn 3d. 
(a)
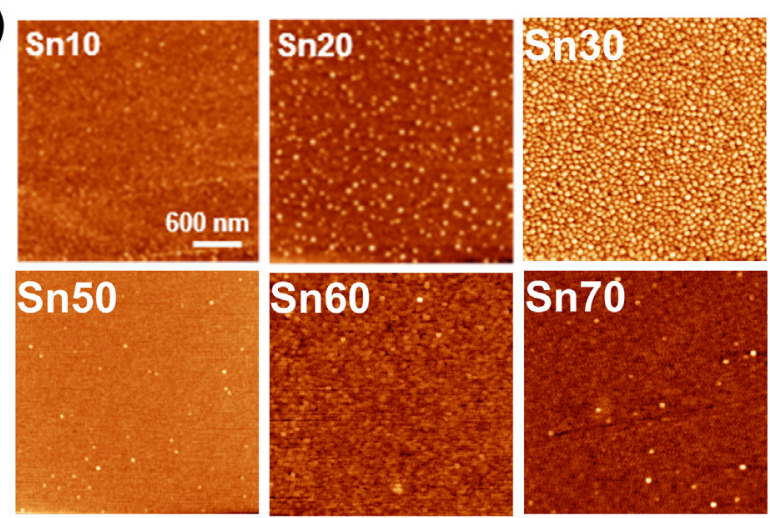

(b)

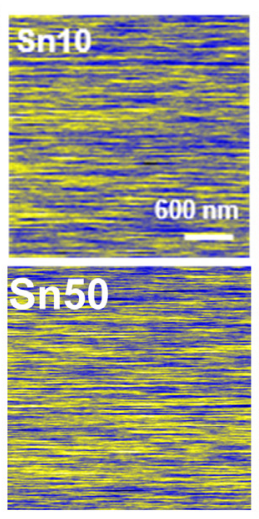

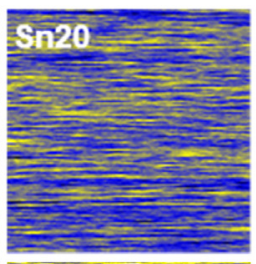
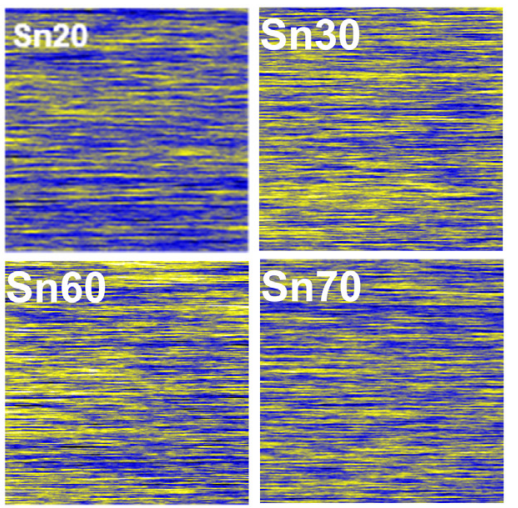

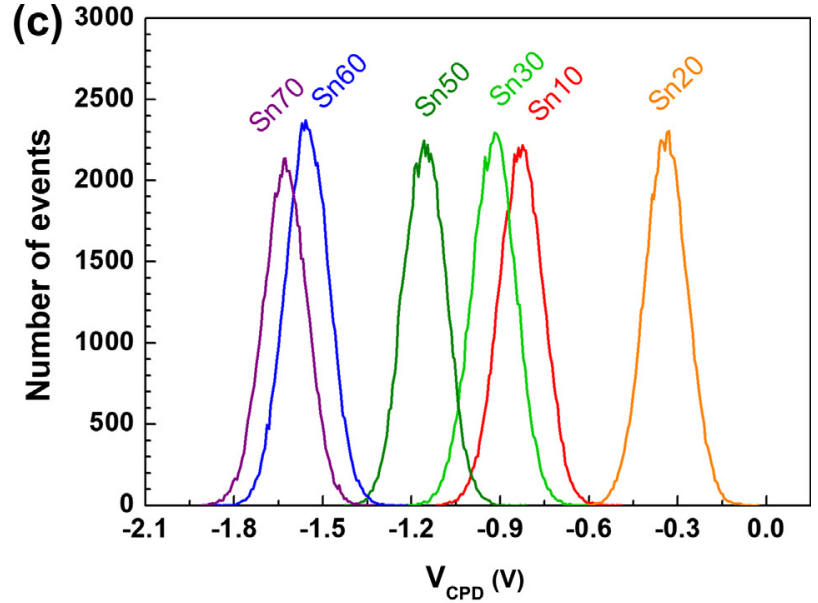

(d)

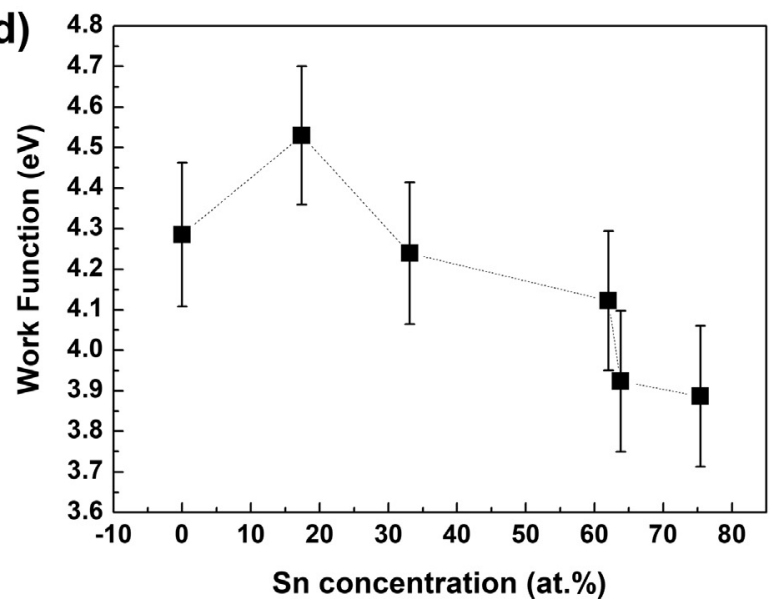

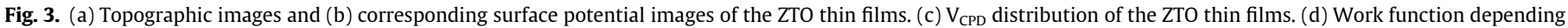
on the $\mathrm{Sn}$ concentration that was calibrated from the $\mathrm{V}_{\mathrm{CPD}}$.

The electrons that were generated from the substitution of the Sn at the $\mathrm{Zn}$ sites formed the major carrier. The substitution occurred easily since the ionic radii of the $\mathrm{Zn}$ and $\mathrm{Sn}$ atoms are similar to each other, as $r\left(\mathrm{Sn}^{4+}\right)=0.69 \AA$ and $\mathrm{r}\left(\mathrm{Zn}^{2+}\right)=0.74 \AA[25,26]$. On the contrary, above the critical point, the $\mathrm{ZnO}$ concentration is lower than the $\mathrm{SnO}_{2}$ concentration, namely $\mathrm{x}<\mathrm{y}$, and as a consequence, free holes formed the major carrier in the ZTO film. It is notable that the n-type carrier concentration is higher than the p-type carrier concentration due to the free electrons that were generated from the oxygen vacancies within the whole $\mathrm{Sn}$ concentration range. In addition, the band edge values such as the conduction band minimum (CBM, $\mathrm{E}_{\mathrm{c}}$ ) and the valence band maximum (VBM, $E_{v}$ ) could be estimated using the carrier concentration that was obtained from the Hall measurement. The semiconductor carrier concentration under the thermal equilibrium can be expressed as follows:

$n=N_{c} \exp \left[-\frac{\left(E_{c}-E_{F}\right)}{k_{B} T}\right]$ and $p=N_{v} \exp \left[\frac{\left(E_{v}-E_{F}\right)}{k_{B} T}\right]$

where $\mathrm{N}_{\mathrm{c}}$ and $\mathrm{N}_{\mathrm{v}}$ are the intrinsic values that are described as $N_{c} \sim N_{v}=2\left(\frac{2 \pi m_{e} k_{B} T}{h^{2}}\right)^{3 / 2}$. The CBM or the VBM of the ZTO thin films with an average carrier concentration, which is based on a Fermi level that is equal to $0 \mathrm{eV}$ is shown in Table 2. With the increasing of the carrier concentration, the ZTO films mobility, which is shown in Fig. 4(b) and was obtained from the division of the Hall coefficient by the resistivity, decreased along with the increasing of the Sn concentration. The electrical conductivity $\sigma$ of the ZTO thin films depends on the carrier concentration $n$ and on their mobility $\mu$ which could be simultaneously measured. The relationship between two parameters is described as $\sigma=\mu n e$ where $\mu=e \tau / m^{*}$. $e$ is the electron charge, $\tau$ is the mean time between collisions, and $m^{*}$ is the effective electron mass. The mobilities of the $n$ - and $p$-type ZTO thin films have more than $130 \mathrm{~cm}^{2} / \mathrm{V} \mathrm{s}$ and $15 \mathrm{~cm}^{2} / \mathrm{V} \mathrm{s}$, respectively. The mobility of the $n$-type ZTO film has a similar value with ITO, whereas the $p$-type ZTO film has a lower value. The conductivity of the ZTO films is affected either by oxygen vacancies or by $\mathrm{Yb}$ dopants. However, since the $\mathrm{Yb}$ content ratio is constant over all the ZTO films, the variation in carrier concentration and mobility with the increase of the Sn content, shown in Fig. 4, is mostly due to the composition ratios of Sn and $\mathrm{Zn}$. The relationship between the mobility and the carrier concentration is attributed to either the ionized impurity scattering or grain boundary scattering [27]. The effect of grain boundary scattering is negligible because the ZTO films have no grain boundary as shown in surface morphologies. Therefore, the mobility of the amorphous ZTO thin film with the lowtemperature growth is mainly dominated by the ionized impurity scattering from $\mathrm{Yb}^{3+}$ or $\mathrm{Sn}^{4+}$.

Fig. 5(a) shows the optical transmission (T) and reflection (R) spectra for the ZTO films consisting of different Sn concentrations 

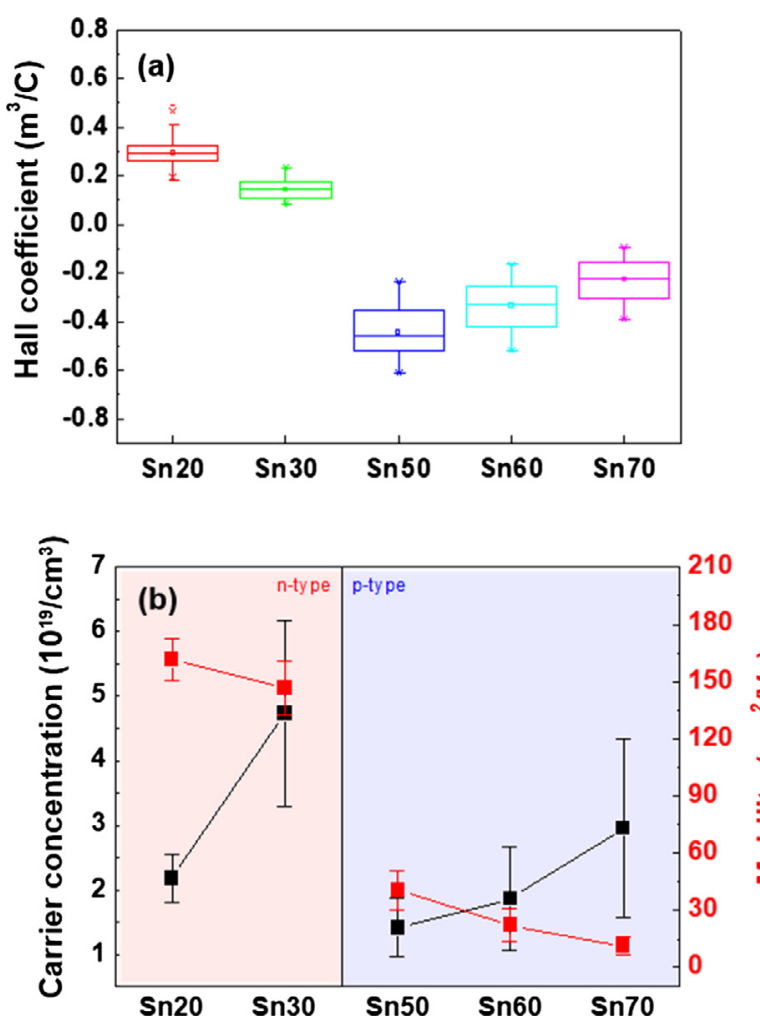

Fig. 4. (a) Distributions of the Hall coefficients and (b) carrier concentrations and mobilities of the ZTO thin films.

Table 2

The average carrier concentration ( $\mathrm{n}$ or $\mathrm{p}$ ) and the CBM or the VBM of the ZTO thin films with different Sn concentrations based on a Fermi level that is equal to $0 \mathrm{eV}$.

\begin{tabular}{lll}
\hline Sn conc. [at.\%] & Carrier concentration $\left[10^{19} \mathrm{~cm}^{-3}\right]$ & Band edge $[\mathrm{eV}]$ \\
\hline 0.0 & $\mathrm{~N} / \mathrm{A}$ & $\mathrm{N} / \mathrm{A}$ \\
17.4 & $\mathrm{n}=2.19$ & $\mathrm{E}_{\mathrm{c}}=+0.35$ \\
33.1 & $\mathrm{n}=4.73$ & $\mathrm{E}_{\mathrm{c}}=+0.33$ \\
62.0 & $\mathrm{p}=1.41$ & $\mathrm{E}_{\mathrm{v}}=-0.36$ \\
63.8 & $\mathrm{p}=1.86$ & $\mathrm{E}_{\mathrm{v}}=-0.35$ \\
75.4 & $\mathrm{p}=2.96$ & $\mathrm{E}_{\mathrm{v}}=-0.34$
\end{tabular}

in the wavelength range from 250 to $1500 \mathrm{~nm}$ using UV-Vis-NIR spectroscopy. The high transparency for all of the ZTO films were observed over $\sim 70 \%$ in the UV-Vis light range. The absorption coefficient ( $\alpha$ ) was calculated from T and R by using $\alpha=\frac{1}{d} \ln \frac{1-R}{T}$, and the optical band gap $\left(E_{g}\right)$ was determined by using the Tauc relation $(\alpha E)^{x}=C\left(h v-E_{g}\right)$, where $\mathrm{h}$ and $v$ are the Planck constant and the photon frequency, respectively. Although non-direct optical transitions are allowed and the exponent $\mathrm{x}$ is 0.5 for the most amorphous semiconductors [28], the direct band gap model provides a sound description of an optical absorption band edge with $x=2$ [29]. Thus, $(\alpha E)^{2}$ is plotted versus the photon energy in Fig. 5 (b) for the ZTO films with different Sn concentrations, in which the $\mathrm{x}$-intercept with the linear fitting yields the optical band gap [30]. Fig. 5(c) shows the variation of the optical band gap with the increasing of the Sn concentration, and this is attributed to the interplay between the Burstein-Moss effect and the renormalization effect [31-33]. The increment of the optical band gap is attributed to the Burstein-Moss effects that results from the increase of the carrier concentration, while the optical band gap was renormalized by the Coulomb repulsion between the added holes in the valence band. A few studies reported that influence of RF (a)
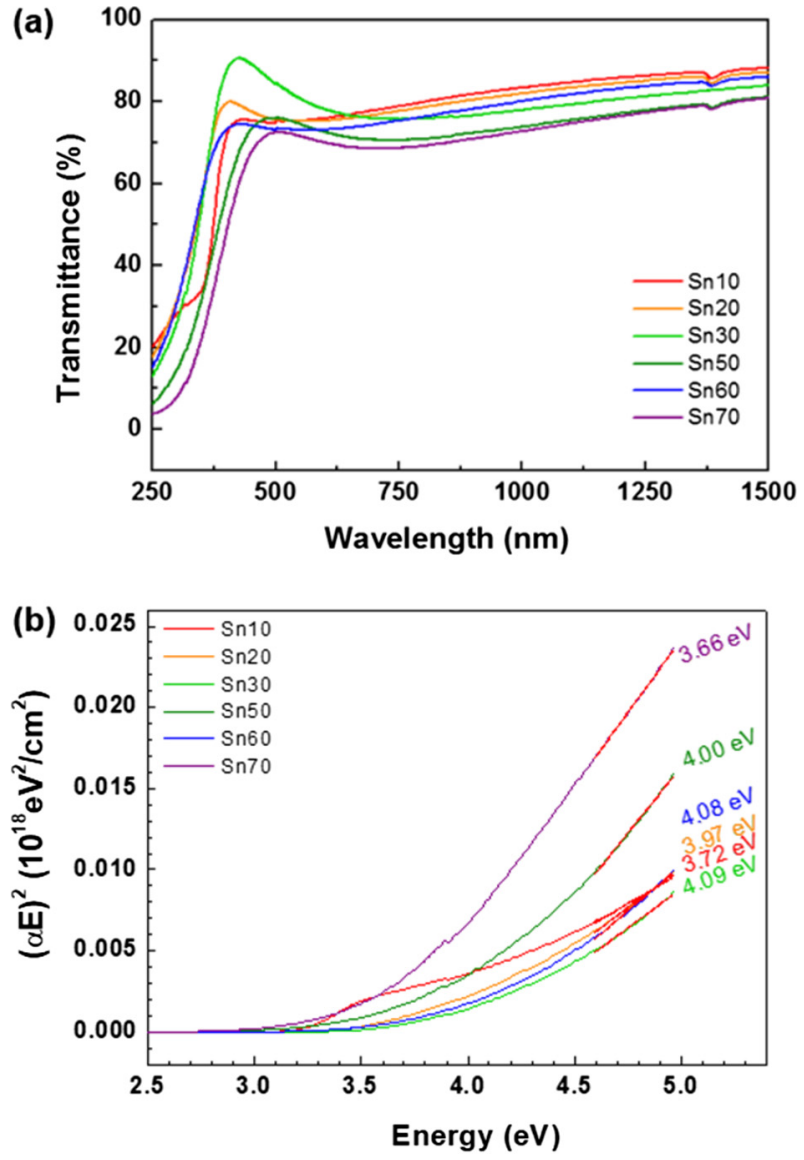

(c)

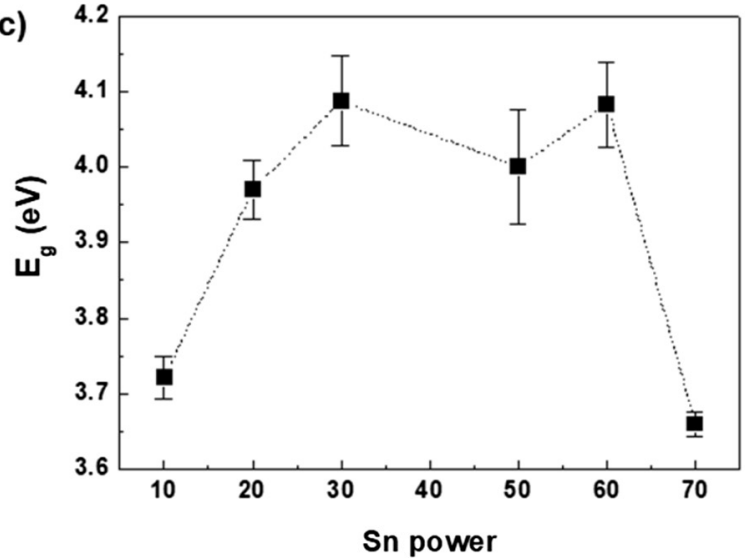

Fig. 5. (a) Transmittance spectra for the ZTO thin films with different Sn concentrations. (b) Extrapolation of the linear part of the $(\alpha \mathrm{E})^{2}$ against the energy $\mathrm{E}$ for the ZTO thin films. (c) Variations of the band gap with the Sn concentration in the ZTO films.

power on bandgap is substantial to make a change of bandgap by $0.05 \mathrm{eV}$ per $150 \mathrm{~W}$ RF power during growth of $\mathrm{ZnO}$ films [34]. Yet, the variation of Sn power in this work is relatively smaller than the previous work.

To extend the previously mentioned results of this section, the CBM, VBM, and vacuum level are shown in Fig. 6 as function of the Sn concentration in ZTO films. The Fermi level has been set to $0 \mathrm{eV}$, and therefore the vacuum level is equal to the work function that was obtained using KPFM. The CBM and VBM were calculated with the carrier concentration and band gap that obtained using the Hall measurement and UV-Vis spectroscopy, respectively. 


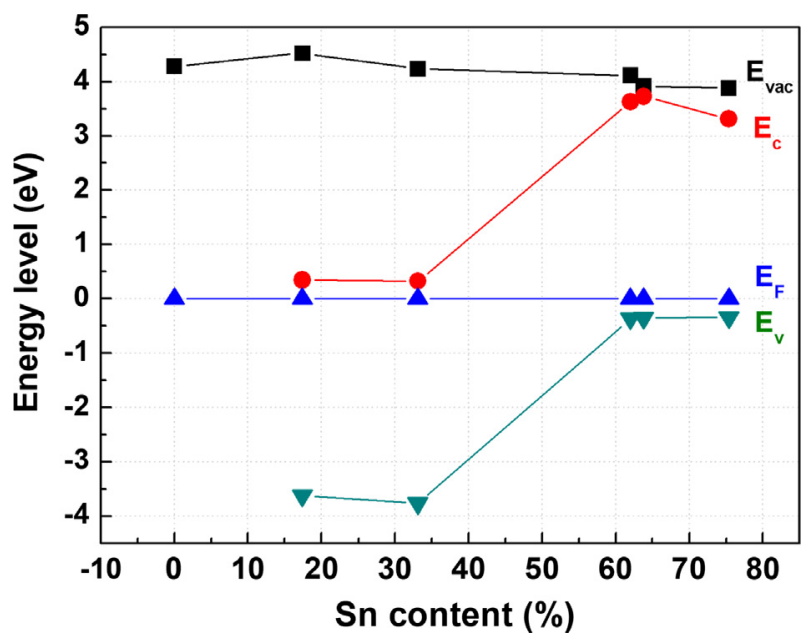

Fig. 6. Variations of the $E_{c}, E_{v}$, and $E_{v a c}$ with the Sn contents in ZTO films.

\section{Conclusion}

The compositional dependence of ZTO thin films on the optical and electrical properties was investigated in this work. The ZTO thin films were prepared using RF magnetron sputtering at different $\mathrm{Sn}$ power levels on glass and p-silicon substrates at a $300{ }^{\circ} \mathrm{C}$ substrate temperature. The amorphous phase in the ZTO thin films was confirmed using symmetric and asymmetric X-ray diffraction $\theta-2 \theta$ scans. The change of the carrier type from the n-type to the $\mathbf{p}-$ type with the increasing of the $\mathrm{Sn}$ concentration was observed through a measurement of the Hall effect because the $\mathrm{Sn}^{4+}$ ions contributed to the generation of the free hole carriers in the ZTO film. The work functions of the ZTO films were measured using KPFM, and they were decreased with increasing of the Sn concentration. The optical transmittance over $70 \%$ in the visible region for all of the ZTO films was observed using the UV-Vis-NIR spectrometer. The optical band gaps of the ZTO films were changed owing to the competition between the Burstein-Moss effect and the renormalization. The Sn dependence of the binding energy was examined according to the measurements of the $\mathrm{Zn} 2 \mathrm{p}$ and Sn 3d XPS spectra. Finally, the band structure that contains the optical band gap, work function, and band edges such as the VBM and the CBM of ZTO thin films was constructed. The results of the present study suggest that the ZTO thin film is competitive compared with indium tin oxide, a representative material of transparent conducting oxides, in terms of optoelectronic devices applications.

\section{Acknowledgments}

This work is supported by the Technology Development Program to solve Climate Changes of the National Research Foundation (NRF) funded by the Ministry of Science, ICT \& Future Planning (2016M1A2A2936753 and 2016M1A2A29366784), by the Basic Science Research Program through the NRF funded by the Ministry of Education (NRF-2017R1D1A1B03034293), and by the DGIST R\&D Program of the Ministry of Science and ICT (17-BD-05). SHO acknowledges that this work was supported by an NRF grant funded by the Korean Government (NRF-2013Fostering Core Leaders of the Future Basic Science Program).

\section{References}

[1] A. Qurashi, M.F. Hossain, M. Faiz, N. Tabet, M.W. Alam, N.K. Reddy, Fabrication of well-aligned and dumbbell-shaped hexagonal $\mathrm{ZnO}$ nanorod arrays and their dye sensitized solar cell applications, J. Alloys Compd. 503 (2010) L40.
[2] F. Jamali-Sheini, K.R. Patil, Dilip S. Joag, Mahendra A. More, Synthesis of Cu$\mathrm{ZnO}$ and $\mathrm{C}-\mathrm{ZnO}$ nanoneedle arrays on zinc foil by low temperature oxidation route: effect of buffer layers on growth, optical and field emission properties, Appl. Surf. Sci. 257 (2011) 8366.

[3] Y.J. Zeng, Z.Z. Ye, W.-Z. Xu, L.-L. Chen, D.-Y. Li, L.-P. Zhu, B.-H. Zhao, Y.-L. Hu, Realization of p-type $\mathrm{ZnO}$ films via monodoping of Li acceptor, J. Cryst. Growth 283 (2005) 180.

[4] W.M. Kim, D.Y. Ku, I. Lee, Y.W. Seo, B.K. Cheong, T.S. Lee, I. Kim, K.S. Lee, The electromagnetic interference shielding effect of indium-zinc oxide/silver alloy multilayered thin films, Thin Solid Films 473 (2005) 315.

[5] G.S. Chae, A modified transparent conducting oxide for flat panel displays only, Jpn. J. Appl. Phys. 40 (2001) 1282.

[6] Y. Nakanishi, K. Kato, M. Horikawa, M. Yonekura, Influence of Zn-Sn ratio on optical property and microstructure of $\mathrm{Zn}-\mathrm{Sn}-\mathrm{O}$ films deposited by magnetron sputtering, Thin Solid Films 612 (2016) 231.

[7] J. Xu, X. Jia, X. Lou, J. Shen, One-step hydrothermal synthesis and gas sensing property of $\mathrm{ZnSnO}_{3}$ microparticles, Solid State Electron. 50 (2006) 504.

[8] W. Körner, C. Elsässer, Density-functional theory study of stability and subgap states of crystalline and amorphous Zn-Sn-O, Thin Solid Films 555 (2014) 81.

[9] V.K. Jain, P. Kumarb, M. Kumarb, P. Jain, D. Bhandarid, Y.K. Vijay, Study of post annealing influence on structural, chemical and electrical properties of ZTO thin films, J. Alloys Compd. 509 (2011) 3541.

[10] C. Jagadish, S.J. Pearton, Zinc Oxide Bulk Thin Films and Nanostructures, Elsevier Science and Technology, Amsterdam, 2006.

[11] S.B. Zhang, S.-H. Wei, A. Zunger, A phenomenological model for systematization and prediction of doping limits in II-VI and I-III-VI 2 compounds, J. Appl. Phys. 83 (1998) 3192.

[12] A. Janotti, C.G. Van de Walle, Fundamentals of zinc oxide as a semiconductor Rep. Prog. Phys. 72 (2009) 126501

[13] L. Li, C.X. Shan, B.H. Li, B. Yao, J.Y. Zhang, D.X. Zhao, Z.Z. Zhang, D.Z. Shen, X.W. Fan, Y.M. Lu, The compensation source in nitrogen doped ZnO, J. Phys. D Appl. Phys. 41 (2008) 245402.

[14] L.J. Van der Pauw, A method of measuring specific resistivity and Hall effect of discs of arbitrary shapes, Philips Res. Rep. 13 (1958) 1.

[15] C.Y. Tsay, H.C. Cheng, Y.T. Tung, W.H. Tuan, C.K. Lin, Effect of Sn-doped on microstructural and optical properties of $\mathrm{ZnO}$ thin films deposited by sol-gel method, Thin Solid Films 517 (2008) 1032

[16] J.F. Moulder, W.F. Stickle, P.E. Sobol, K.D. Bomben, Handbook of X-ray Photoelectron Spectroscopy Physical Electronics, Eden Prairie, Minnesota 1995.

[17] M.N. Islam, T.B. Ghosh, K.L. Chopra, H.N. Acharya, XPS and X-ray diffraction studies of aluminum-doped zinc oxide transparent conducting films, Thin Solid Films 280 (1996) 20

[18] O.D. Jayakumar, C. Sudakar, A. Vinu, A. Asthana, A.K. Tyagi, Effect of surfactant treatment on the magnetic properties of Mn-doped $\mathrm{ZnO}$ bulk and nanoparticles, J. Phys. Chem. C 113 (2009) 4814.

[19] A. Bougrine, M. Addou, A. Kachouane, J.C. Bernède, M. Morsli, Effect of tin incorporation on physicochemical properties of $\mathrm{ZnO}$ films prepared by spray pyrolysis, Mater. Chem. Phys. 91 (2005) 247.

[20] M. Kwoka, L. Ottaviano, M. Passacantando, S. Santucci, G. Czempik, J. Szuber XPS study of the surface chemistry of L-CVD $\mathrm{SnO}_{2}$ thin films after oxidation, Thin Solid Films 490 (2005) 36.

[21] Z. Pan, X. Tian, S. Wu, C. Xiao, Z. Li, J. Deng, G. Hu, Z. Wei, Effects of Al and Sn dopants on the structural and optical properties of $\mathrm{ZnO}$ thin films, Superlattice. Microstruct. 54 (2013) 107.

[22] J.H. Ko, I.H. Kim, D. Kim, K.S. Lee, T.S. Lee, B. Cheong, W.M. Kim, Transparent and conducting $\mathrm{Zn}-\mathrm{Sn}-\mathrm{O}$ thin films prepared by combinatorial approach, Appl. Surf. Sci. 253 (2007) 7398.

[23] W. Melitz, J. Shen, A.C. Kummel, S. Lee, Kelvin probe force microscopy and its application, Surf. Sci. Rep. 66 (2011) 1.

[24] F.A. Kroger, H.J. Vink, Relations between the concentrations of imperfections in crystalline solids, Solid State Phys. 3 (1956) 367.

[25] S. Ilican, M. Caglar, Y. Caglar, Y. Sn doping effects on the electro-optical properties of sol gel derived transparent ZnO films, Appl. Surf. Sci 256 (2010) 7204.

[26] X. Yan, D. Hu, H. Li, L. Li, X. Chong, Y. Wang, Nanostructure and optical properties of $\mathrm{M}$ doped $\mathrm{ZnO}(\mathrm{M}=\mathrm{Ni}, \mathrm{Mn})$ thin films prepared by sol-gel process, Phys. B: Condens. Matter 406 (2011) 3956.

[27] H.L. Hartnagel, A.L. Dawar, A.K. Jain, C. Jagadish, Semiconducting Transparent Thin Films, Institute of Physics, Bristol, 1995.

[28] N.F. Mott, E.A. Davis, Electronic Processes in Non-crystalline Materials, Oxford University Press, Oxford, 1979.

[29] K. Saravanakumar, K. Ravichandran, Synthesis of heavily doped nanocrystalline $\mathrm{ZnO}$ : Al powders using a simple soft chemical method, J. Mater. Sci. Mater. Electron. 23 (2012) 1462.

[30] J.I. Pankove, Optical Processes in Semiconductors, Dover Publications, New York, 1971.

[31] T.S. Moss, The interpretation of the properties of indium antimonide, Proc Phys. Soc. B 67 (1954) 775.

[32] E. Burstein, Anomalous optical absorption limit in InSb, Phys. Rev. 93 (1954) 632.

[33] K.-F. Berggren, B.E. Sernelius, Band-gap narrowing in heavily doped manyvalley semiconductors, Phys. Rev. B 24 (1981) 1971.

[34] Y.M. Hu, J.Y. Li, N.Y. Chen, C.Y. Chen, T.C. Han, C.C. Yu, Effect of sputtering power on crystallinity, intrinsic defects, and optical and electrical properties of Al-doped $\mathrm{ZnO}$ transparent conducting thin films for optoelectronic devices, J. Appl. Phys. 121 (2017) 085302. 